\title{
Pancreatic carcinoma and diabetes mellitus
}

\author{
Abstract \\ Pancreatic carcinoma $(\mathrm{PaC})$ is a rare disease with one of the highest mortality rates and a \\ continuously increasing incidence. Surgery is the only possibility as a curative treatment, \\ but, unfortunately, the tumor is often diagnosed in an inoperative stage because of its \\ asymptomatic/aspecific progression. Until now, there is no feasible screening method for \\ early-stage sporadic $\mathrm{PaC}$. This article aims to review the connection between $\mathrm{PaC}$ and \\ diabetes mellitus (DM), the potential screening group for $\mathrm{PaC}$; to investigate the possibility \\ of differentiating $\mathrm{PaC}$-associated $\mathrm{DM}(\mathrm{PaCDM})$ from type 2 diabetes mellitus (T2DM); \\ and to summarize the effect of metformin on $\mathrm{PaC}$ based on the results of the latest medical \\ publications.
}

Volume 6 Issue 4 - 2017

\section{Dora Illes, Laszlo Czako}

Department of Medicine, University of Szeged, Hungary

Correspondence: Laszlo Czako, Department of Medicine, University of Szeged, Hungary,

Email czako.laszlo@med.u-szeged.hu

Received: January 18, 2017 | Published: March 23, 2017

Keywords: pancreatic carcinoma, diabetes mellitus, screening, metformin

\section{Introduction}

$\mathrm{PaC}$ accounts for only $3 \%$ of all cancer cases, ${ }^{1}$ with a continuously increasing incidence. ${ }^{2}$ Hungary is in the third place in Europe based on the incidence $(10-15 / 100,000$ persons per year) and prevalence of $\mathrm{PaC}{ }^{3}$ The Central European region has the highest mortality rate from $\mathrm{PaC}$ in Europe. ${ }^{4} \mathrm{PaC}$ is the third leading cause of cancer-related death in the USA. ${ }^{5}$

The prognosis for $\mathrm{PaC}$ is extremely poor: it has the lowest five-year survival of all cancers, only $6 \%,{ }^{6}$ and this rate has not changed during the last 40years. ${ }^{7}$ It depends on the late diagnosis of the disease: in the presence of the aspecific symptoms, $\mathrm{PaC}$ is often in an advanced stage, which means that the possibility of a curative surgical intervention is low. Screening $\mathrm{PaC}$ in an asymptomatic stage is recommended for a better outcome. ${ }^{8}$ Population-wide screening is not feasible because the lifetime prevalence of $\mathrm{PaC}$ is low, only $1.39 \%{ }^{9}$ In fact, screening of individuals under 70 who have a lifetime risk of $\mathrm{PaC}$ of $16 \%$ or greater is cost-effective. ${ }^{10}$

\section{Connection between pancreatic cancer and diabetes mellitus}

The connection between $\mathrm{PaC}$ and $\mathrm{DM}$ has been well known for decades. ${ }^{11}$ Among risk conditions (such as hereditary pancreatitis or multiorgan cancer syndromes and a positive family history of $\mathrm{PaC}$; Table 1. DM has the strongest link to PaC: $40-65 \%$ of pancreatic cancer patients meet the criteria for $\mathrm{DM},{ }^{12}$ in contrast to these genetic factors, whose role in $\mathrm{PaC}$ is less than $10 \%$ (Lee 2,3). Based on a prospective study, the rate of $\mathrm{DM}$ among $\mathrm{PaC}$ patients is higher than in the normal population: in nearly $50 \%$ of $\mathrm{PaC}$ cases, $\mathrm{DM}$ was present as new-onset or concomitant at the time of the cancer diagnosis. ${ }^{13}$ Retrospective studies with a huge number of cases showed that longterm DM and resultant hyperinsulinemia pose 2.17times the risk for developing $\mathrm{PaC}^{14}$ through the effect of insulin as a growth factor and the elevated level of mitogen cell proliferation-enhancing insulin-like growth factor-1 (IGF-1). DM could be not only a cause, but also a consequence of the tumor: the new-onset $(<36$ month) DM patients have an eightfold risk of contracting $\mathrm{PaC}$ within three years from the time of diagnosis of DM. ${ }^{15}$ Based on the temporal relationship, two groups can be distinguished: in one, early-onset, long-term DM is the cause of $\mathrm{PaC}$, and in the other, late-onset, short-term $\mathrm{DM}$ is the consequence of $\mathrm{PaC} .{ }^{16}$ The definition of new-onset $\mathrm{DM}$ has recently been changed: instead of 36months, DM identified 24 months before $\mathrm{PaC}$ diagnosis is called new-onset DM. ${ }^{17}$ It is not cost-effective to screen patients with long-term $\mathrm{DM}$ for $\mathrm{PaC} .{ }^{8}$ It is known that only $1 \%$ of newly diagnosed $\mathrm{DM}$ patients over 50 develop $\mathrm{PaC}$ within three years from the onset of $\mathrm{DM},{ }^{10}$ but in these cases the tumor is often resectable. ${ }^{18}$ We proved in our study that patients with new-onset DM constitute a feasible risk group for $\mathrm{PaC}$ screening. Unfortunately, we could not screen any early-stage $\mathrm{PaC}$ either with an imaging tool or an elevated level of tumor marker carbohydrate antigen 19-9. ${ }^{19}$ Therefore, it is recommended that the tumor-specific differences and clinical manifestations of $\mathrm{PaC}$ be investigated for effective screening of early-stage tumors instead of doing instrumental examinations.

\section{PaC: Pancreatic Carcinoma}

The differentiation of PaCDM from "traditional" T2DM plays a key role in the screening method, thus leading to a number of studies that investigate this question. One of the relevant differences is the change in body weight. In PaCDM cases, patients lost weight before the onset of DM and continued losing weight despite antidiabetic therapy until they were diagnosed with $\mathrm{PaC}$ as compared to T2DM patients, who gained weight even after adequate DM therapy was implemented. Weight loss appeared earlier in PaCDM than other PaC symptoms (abdominal pain, fatigue and anorexia), evidence that it is not a consequence of cachectization. It has been proposed that weight loss results from overproduction of a "lipid mobilizing factor" zinkalpha-2-glycoprotein (ZAG) and resultant fatty acid mobilization. In the case of $\mathrm{PaCDM}$, the escalation of antidiabetic therapy is required parallel to the weight loss, unlike in T2DM cases..$^{20}$ The investigation by Lee et al. strengthens the findings above with additional alarm signs: PaCDM patients were older and had more weight loss, lower premorbid $\mathrm{BMI}$, more family history of $\mathrm{PaC}$ and less family history of DM compared to the new-onset T2DM patients. With regard to insulin resistance (IR), the two groups exhibited further differences, which are confirmed by the homeostatic model assessment index: IR is lower in PaCDM than in $\mathrm{T} 2 \mathrm{DM},{ }^{21}$ and its level is similar to that of the normal healthy population. ${ }^{22}$ Unfortunately, it is not sufficient to find a relatively small subgroup based on clinical manifestations of tumors eligible for screening if we cannot precisely differentiate between ill and healthy individuals. Because of the ineffectiveness of imaging tools and tumor markers for screening, the investigation of biomarkers came into view. It has been proved that there is a disparity between PaCDM and T2DM in the serum levels of neuroendocrine 
mediators: the mean plasma level of leptin, pancreatic polypeptide (PP) and glucose-dependent insulinotropic peptide (GIP) is significantly lower in PaCDM than that in T2DM, and the level of adiponectin is higher. This significance is more explicit if the PaCDM group is further restricted to patients with new-onset DM and $>2 \mathrm{~kg}$ weight loss compared to the "simple" T2DM cases. ${ }^{22}$ The lower serum levels of GIP and PP were present among PaC patients with normal glucose tolerance, suggesting that these findings are rather the consequence of $\mathrm{PaC}$ only. Leptin increases cell proliferation, migration and tumor cell invasion, while adiponectin inhibits cell growth, invasion and tumor progression through stimulation of tumor cell apoptosis. ${ }^{23}$ These facts suggest that the lower level of leptin and the higher level of adiponectin are a compensatory response of the human body itself to the tumor process. Škrha et al., ${ }^{22}$ found these differences in advanced-stage $\mathrm{PaC}$ cases, thus confirming this theory. One of the limitations of their study is that they lack data from early-stage $\mathrm{PaC}$ cases. It remains unclear whether the divergence in serum levels of neuroendocrine mediators is appropriate for the screening of earlystage $\mathrm{PaC}$. Most of the studies suggest that $\mathrm{PaCDM}$ is a paraneoplastic sign caused by tumor-produced factors, such as adrenomedullin, a potential mediator of $\beta$-cell dysfunction in pancreatic cancer-induced diabetes, and an increased expression of proteases, such as fibroblast activation protein alpha and dipeptidyl peptidase 4 , which can cause a lower GIP level in PaC. In a study by Basso et al., daily intraperitoneal injection of supernatant from pancreatic cancer cell line MIA PaCa2 into immunodeficient mice led to a significant increase in blood glucose levels and significantly reduced glucose tolerance compared to control mice injected with saline. The 14 amino acid peptide from S100A8 impairs the catabolism of glucose with myoblasts in vitro and may cause hyperglycemia in vivo. ${ }^{17,22,24-26}$ The investigation of complex connections between $\mathrm{PaC}$ and $\mathrm{DM}$ resulted in an important question: does antidiabetic therapy influence the tumor development/ process, and if so, how?

Table I Clinical conditions with elevated risk for pancreatic carcinoma (responsible gene) and the relative risk

\begin{tabular}{lll}
\hline Clinical Conditions & Relative Risk (x) & Responsible Gene \\
\hline Smoking & 2.5 & \\
Chronic pancreatitis & 15 & \\
Diabetes mellitus & 2.2 & \\
Obesity & 1.2 & \\
Peutz-Jeghers syndrome & 132 & STKII/LKBI \\
Hereditary atypical multiple mole melanoma & $20-47$ & CDKN2A \\
Hereditary breast/ovarium cancer & $3-10$ & BRCA2 \\
Hereditary non-polypotic colorectal carcinoma & 9 & MLHI,MSH2,MSH6,PMS2 \\
Familial adenomatosus polyposis & 4 & APC \\
Fanconi anemia & - & PALB2 \\
Ataxia teleangiectasia & 3 & ATM \\
Li-Fraumeni syndrome & 7 & P53 \\
Hereditary pancreatitis & $50-80$ & PRSSI/SPINKI \\
Cystic fibrosis & 5 & CFTR \\
3<First relative with PaC & 32 & \\
2 First relative with PaC & 6.4 & \\
I First relative with PaC & 4.5 & \\
\hline
\end{tabular}

\section{Metformin and pancreatic cancer}

Long-term DM increases the risk of developing $\mathrm{PaC}$ through hyperinsulinemia and overexpression of insulin and insulin-like growth factor-1 (IGF-1) receptors. ${ }^{27}$. The "first choice" antidiabetic in T2DM, metformin interacts with the signaling pathway of insulin and IGF- $1 .{ }^{28}$ Metformin operates through the activation of adenosine monophosphate- activated protein kinase (AMPK), which leads to the inhibition of the mammalian target of rapamycin (mTOR), stops the insulin/IGF-1 pathway and results in the inhibition of their mitotic effects and tumor progression. The inhibition of mTOR decreases protein synthesis and the intensity of cell growth, processes which play an important role in survival. AMPK promotes the function of tumor suppressor p53 and reduces the serum levels of insulin and IGF-1. ${ }^{29}$ Some studies showed that metformin can sensitize cancer cells to both chemotherapy ${ }^{30,31}$ and radiotherapy. ${ }^{32,33}$ Metformin is increasingly accepted as an antitumor agent. It can lessen the risk of T2DM patients developing $\mathrm{PaC}$ if used continuously over a long period: a meta-analysis based on 11 studies showed that using metformin lowered the risk of $\mathrm{PaC}$ by $37 \%$ compared to other antidiabetics. ${ }^{34}$ It influences the survival of PaCDM patients as an independent predictor of improved outcome in this group. The twoyear survival was $30 \%$ in the metformin group compared to $15 \%$ in the non-metformin group among $\mathrm{PaC}$ patients. ${ }^{35}$ Metformin can improve survival even in the case of advanced-stage $\mathrm{PaC}$ treated with palliative chemotherapy compared to the non-diabetic $\mathrm{PaC}$ patients not taking metformin (overall survival was 11 months, 7.5 months and 7.9 months in these groups, respectively). The only limitation of this drug is that its positive effects do not prevail if metastases are present. ${ }^{36}$

\section{Summary}

The connection between $\mathrm{PaC}$ and $\mathrm{DM}$ is complex and bidirectional. Screening for early-stage $\mathrm{PaC}$ is recommended for high-risk group patients with new-onset DM who present with the following alarm signs: old age (>55years) (19), low/normal BMI at the time of DM diagnosis, antidiabetic therapy-resistant weight loss, and PaC-positive and DM-negative family history. The low level of insulin resistance may be a potential differentiating factor between PaCDM and T2DM. The search for biomarkers that are specific only to PaC, thus making early stage cancer screening possible, is still in progress. The effect of metformin in tumor prevention and in survival improvement has 
been confirmed by numerous studies. It is important to emphasize that these effects are present not only in diabetic patients, but also in nondiabetics. Thus, in the case of non-metastatic pancreatic cancer, it is recommended that metformin be integrated into the therapy.

\section{Acknowledgments}

None.

\section{Conflicts of interest}

Author declares there are no conflicts of interest.

\section{Funding}

None.

\section{References}

1. Loc WS, Smith JP, Matters G, et al.Novel strategies for managing pancreatic cancer. World J Gastroenterol. 2014;20(40):14717-14725

2. Siegel R, Ma J, Zou Z, et al.Cancer Statistics. CA Cancer J Clin. 2014;64(1):9-29.

3. Szmola R, Farkas G, Hegyi P, et al. Pancreatic cancer. Evidence based management guidelines of the Hungarian Pancreatic Study Group. Orv Hetil. 2015;156(8):326-339.

4. Hariharan D, Saied A Kocher HM. Analysis of mortality rates for pancreatic cancer across the world. HPB (Oxford). 2008;10(1):58-62.

5. Cancer Statistics Center. American Cancer Society. 2017.

6. Ferlay J. GLOBOCAN 2012 v1.0, Cancer Incidence and Mortality Worldwide: IARC Cancer Base No. 11 [Internet]. International Agency for Research on Cancer, Lyon, France. 2013.

7. Siegel R, Ma J, Zou Z, et al.Cancer statistics. CA Cancer J Clin. 2014;64(1):9-29

8. Chari ST, Kelly K, Hollingsworth MA, et al. Early detection of sporadic pancreatic cancer: summative review. Pancreas. 2015;44(5):693-712.

9. National Cancer Institue. SEER cancer statistics review 1975-2006.

10. Chari ST. Detecting early pancreatic cancer: problems and prospects. Semin Onco. 2007;134(4):284-294

11. Aggarwal G, Rabe KG, Petersen GM, et al.New-onset diabetes in pancreatic cancer: a study in the primary care setting. Pancreatology. 2012;12(2):156-161.

12. Ben Q, Cai Q, Li Z, et al.The relationship between new-onset diabetes mellitus and pancreatic cancer risk: a case-control study. Eur J Cancer. 2011;47(2):248-254.

13. Dugnani E, Gandolfi A, Balzano G, et al. Diabetes associated with pancreatic ductal adenocarcinoma is just diabetes: Results of a prospective observational study in surgical patients. Pancreatology. 2016;16(5):844-852.

14. A Yacoub, E Siegel, I Makhoul. Pancreatic cancer and diabetes mellitus: A retrospective cohort study. J Clin Oncol. 2011;29(15_suppl):4102.

15. Chari ST, Leibson CL, Rabe KG, et al. Probability of pancreatic cancer following diabetes: A population-based study. Gastroenterology. 2005;129(2):504-511.

16. Mizuno S, Nakai Y, Isayama $\mathrm{H}$, et al. Risk factors and early signs of pancreatic cancer in diabetes: screening strategy based on diabetes onset age. J Gastroenterol. 2013;48(2):238-246.

17. Sah RP, Nagpal SJ, Mukhopadhyay D, et al. New insights into pancreatic cancer-induced paraneoplastic diabetes. Nat Rev Gastroenterol Hepatol. 2013;10(7):423-433.
18. Pelaez-Luna M, Takahashi N, Fletcher JG, et al.Resectability of presymptomatic pancreatic cancer and its relationship to onset of diabetes: a retrospective review of CT scans and fasting glucose values prior to diagnosis. Am J Gastroenterol. 2007;102(10):2157-2163

19. Illes D, Terzin V, Holzinger G, et al. New-onset type 2 diabetes mellitus-A high-risk group suitable for the screening of pancreatic cancer? Pancreatology. 2016;16(2):266-271.

20. Hart PA, Kamada P, Rabe KG, et al. Weight loss precedes cancer-specific symptoms in pancreatic cancer-associated diabetes mellitus. Pancreas. 2011;40(5):768-772.

21. Lee JH, Kim SA, Park HY, et al. New-onset diabetes patients need pancreatic cancer screening? J Clin Gastroenterol . 2012;46(7):e58-61.

22. Skrha J, Busek P, Uhrova J, et al. Lower plasma levels of glucosedependent insulinotropic peptide (GIP) and pancreatic polypeptide (PP) in patients with ductal adenocarcinoma of the pancreas and their relation to the presence of impaired glucoregulation and weight loss. Pancreatology. 2016;17(1):89-94.

23. Michael N, VanSaun. Molecular pathways: Adiponectin and leptin signaling in cancer. Clin Cancer Res. 2013;19(8):1926-1932.

24. Pannala R, Basu A, Petersen GM, et al.New-onset diabetes: a potential clue to the early diagnosis of pancreatic cancer. Lancet Oncol. 2009;10(1):88-95

25. Basso D, Greco E, Fogar P, et al. Pancreatic cancer-derived S-100A8 N-terminal peptide: a diabetes cause? Clin Chim Acta. 2006;372(12):120-128.

26. Andersen DK, Andren-Sandberg Å, Duell EJ, et al. Pancreatitis diabetes - pancreatic cancer: summary of an NIDDK-NCI workshop. Pancreas. 2013;42(8):1227-1237

27. Pollak M. Insulin and insulin-like growth factor signaling in neoplasia. Nat Rev Cancer. 2008;8(12):915-928.

28. Rosengurt E, Sinnett-Smith J, Kisfalvi K. Cross-talk between insulin insulin-like growth factor-1 receptors and $\mathrm{G}$ protein-coupled receptor signaling systems: a novel target for the antidiabetic drug metformin in pancreatic cancer. Clin Cancer Res. 2010;16(9):2505-2511.

29. Jun Gong, Lori A Robbins, Aurelia Lugea, et al. Diabetes, pancreatic cancer, and metformin therapy. Front Physiol. 2014;5:426

30. Dong L, Zhou Q, Zhang Z, et al. Metformin sensitizes endometrial cancer cells to chemotherapy by repressing glyoxalase I expression. $J$ Obstet Gynaecol Res. 2012;38(8):1077-1085.

31. Kawanami T, Takiguchi S, Ikeda N, et al.A humanized anti-IGF1R monoclonal antibody (R1507) and/or metformin enhance gemcitabine-induced apoptosis in pancreatic cancer cells. Oncol Rep. 2012;27(3):867-872.

32. Song CW, Lee H, Dings RP, et al. Metformin kills and radiosensitizes cancer cells and preferentially kills cancer stem cells. Sci Rep. 2012;2:362.

33. Storozhuk Y, Hopmans SN, Sanli T, et al. Metformin inhibits growth and enhances radiation response of non-small cell lung cancer (NSCLC) through ATM and AMPK. Br J Cancer. 2013;108(10):2021-2032.

34. Wang Z, Lai ST, Xie L, et al. Metformin is associated with reduced risk of pancreatic cancer in patients with type 2 diabetes mellitus: a systematic review and meta-analysis. Diabetes Res Clin Pract. 2014;106(1):19-26.

35. Sadeghi N, Abbruzzese JL, Yeung SC, et al.Metformin Use is Associated with Better Survival of Diabetic Patients with Pancreatic Cancer. Clin Cancer Res. 2012;18(10):2905-2912.

36. Choi Y, Kim TY, Oh DY, et al. The Impact of Diabetes Mellitus and Metformin Treatment on Survival of Patients with Advanced Pancreatic Cancer Undergoing Chemotherapy. Cancer Res Treat. 2016;48(1):171179 